\title{
Morbus Parkinson - verzögert freigesetztes Amantadin reduziert Dyskinesien
}

\begin{abstract}
Mit einer neu entwickelten, verzögert freigesetzten Amantadin-Formulierung lassen sich Dyskinesien bei Morbus Parkinson offenbar gut lindern. Darauf deuten Ergebnisse einer Pilotstudie mit 121 Patienten.
\end{abstract}

auf der Unifield Dyskinesia Rating Scale (UDysRS, maximal 104 Punkte).

Die Patienten waren im Schnitt 65 Jahre alt, seit neun Jahren an Parkinson erkrankt und benötigten seit etwas mehr als sieben Jahren L-Dopa. Die Tagesdosis lag mittlerweile bei rund $860 \mathrm{mg}$.

\section{Dyskinesien um $40 \%$ reduziert}

Zu Beginn der Therapie lag der Wert auf der Dyskinesieskala bei rund 40 Punkten. Mit der Amantadin-Formulierung ging er rasch (innerhalb von zwei Wochen) um rund 15 Punkte zurück und blieb dann konstant. Unter Placebo sank der Wert lediglich um 6 Punkte. Nach zwölf Wochen lag die Differenz zwischen Placebo und Amantadin bei 7,9 Punkten, zum Studienende bei 9,3 Punkten ( $p=0,0008)$. Mit Amantadin sank der Wert auf der Dyskinesieskala innerhalb von 24 Wochen um $40 \%$, mit Placebo um $16 \%$.

Nach zwölf Wochen war zudem die Zeit mit behindernden Dyskinesien in der Amantadin-Gruppe um 3,2 Stunden und in der Placebogruppe um 1,6 Stunden pro Tag zurückgegangen, zum Studienende ließ sich eine Reduktion von je 3,3 und 1,9 Stunden beobachten. OffPhasen gingen unter Amantadin in den ersten drei Monaten um 0,6 Stunden zurück, mit Placebo nahmen sie um 0,3 Stunden zu, auch dieses Ergebnis veränderte sich kaum bis zum Therapieende. Schließlich verlängerte sich die dyskinesiefreie On-Zeit mit ADS-5102 um 3,6 Stunden (Placebo: 0,9 Stunden).

Mit der Amantadin-Formulierung verbesserten sich der klinische Gesamteindruck sowie der Wert auf der UPDRS-Parkinson-Skala (Part IV) signifikant gegenüber Placebo. Die Schlafdauer wurde hingegen nicht beeinträchtigt.

\section{Halluzinationen, Ödeme, Stürze}

Als Nebenwirkungen traten unter Amantadin vermehrt Halluzinationen, periphere Ödeme und Benommenheit auf (bei jeweils rund $25 \%$ der Patienten), auch Verstopfung und Stürze (bei jeweils $16 \%)$ wurden häufiger beobachtet. Die Halluzinationen wurden vor allem im ersten Therapiemonat beobachtet und führten nicht zum Therapieabbruch. Insgesamt $21 \%$ der Patienten unter Amantadin und $7 \%$ unter Placebo brachen die Therapie wegen unerwünschter Wirkungen ab.

Die Forscher um Pahwa stellen eine rasche und anhaltende antidyskinetische Wirkung der neuen Formulierung fest. Das Präparat wird derzeit vom Unternehmen Adamas Pharmaceuticals entwickelt.

Thomas Müller, Springer Medizin

Emerging Science Session. Pahwa R et al. ADS5102 (amantadine- $\mathrm{HCl}$ ) extended-release capsule reduced levodopa-induced dyskinesia (LID) in the phase 3 EASE LID study. 68. AAN-Kongress, Vancouver, 15.-21.4.2016

\section{Neuer Wirkstoff lindert Spätdyskinesien}

\section{Eine Behandlung mit Valbenazin reduziert Neuroleptika-induzierte Spätdyskinesien. In einer Phase-III-Studie gingen die Beschwerden um etwa ein Drittel zurück.}

$S^{p}$ pätdyskinesien mit Zuckungen und abnormalen Bewegungen von Zunge, Lippen, Kiefern sowie Extremitäten sind keine Seltenheit nach langjähriger Dopaminrezeptorblockade, wie sie bei den meisten Patienten mit Schizophrenie nötig ist. Häufig persistieren die Symptome auch nach dem Absetzen der Medikation, wirksame antidyskinetische Therapien sind bisher nicht zugelassen.

Ärzte um Dr. Robert Hauser von der Universität in Tampa, Florida, stellten Daten einer Phase-III-Studie mit dem Wirkstoff Valbenazin vor. Die Substanz blockiert den präsynaptischen vesikulären Monoamintransporter Typ 2 (VMAT2) und senkt damit reversibel die Dopaminfreisetzung an den Nervenendigungen. D2-Dopaminrezeptoren und der VMAT1 sind nicht betroffen, was unerwünschte Wirkungen in anderen, nicht für Dyskinesien ursächlichen dopaminergen Systembereichen verhindern soll. „Die Substanz hat auch ein günstiges pharmakokinetisches Profil und ermöglicht eine einmal tägliche Einnahme ohne Auftitrieren“, erläuterte Hauser. 